\title{
First Implementation of TES Bolometer Arrays with SQUID-based Multiplexed Readout on a Balloon-Borne Platform
}

François Aubin ${ }^{a}$, Asad M. Aboobaker ${ }^{b}$, Peter Ade ${ }^{c}$, Carlo Baccigalupi ${ }^{d}$, Chaoyun Bao ${ }^{b}$, Julian Borrill $^{e}$, Christopher Cantalupo ${ }^{e}$, Daniel Chapman ${ }^{f}$, Joy Didier $^{f}$, Matt Dobbs $^{a}$, Will Grainger ${ }^{c}$ , Shaul Hanany ${ }^{b}$, Johannes Hubmayr ${ }^{g}$, Peter Hyland ${ }^{a}$, Seth Hillbrand ${ }^{f}$, Andrew Jaffe ${ }^{h}$, Bradley Johnson $^{i}$, Terry Jones ${ }^{b}$, Theodore Kisner ${ }^{e}$, Jeff Klein ${ }^{b}$, Andrei Korotkov ${ }^{j}$, Sam Leach $^{d}$, Adrian

Lee $^{i}$, Michele Limon ${ }^{f}$, Kevin MacDermid ${ }^{a}$, Tomotake Matsumura ${ }^{k}$, Xiaofan Meng ${ }^{i}$, Amber

Miller $^{f}$, Michael Milligan ${ }^{b}$, Daniel Polsgrove ${ }^{b}$, Nicolas Ponthieu $^{l}$, Kate Raach ${ }^{b}$, Britt

Reichborn-Kjennerud $^{f}$, Ilan Sagiv ${ }^{b}$, Graeme Smecher ${ }^{a}$, Huan Tran ${ }^{i}$, Gregory S. Tucker ${ }^{j}$, Yury

Vinokurov $^{j}$, Amit Yadav ${ }^{m}$, Matias Zaldarriaga ${ }^{m}$ and Kyle Zilic ${ }^{b}$

${ }^{a}$ McGill University, Montréal, Quebec, H3A 2T8, Canada;

${ }^{b}$ University of Minnesota School of Physics and Astronomy, Minneapolis, MN 55455;

${ }^{c}$ Cardiff University, Cardiff, CF24 3AA, United Kingdom;

${ }^{d}$ Scuola Internazionale Superiore di Studi Avanzati, Trieste 34151, Italy;

${ }^{e}$ Lawrence Berkeley National Laboratory, Berkeley, CA 94720;

${ }^{f}$ Columbia University, New York, NY 10027;

${ }^{g}$ National Institute of Standards and Technology, Boulder CO 80305;

${ }^{h}$ Imperial College, London, SW7 2AZ, England, United Kingdom;

${ }^{i}$ University of California, Berkeley, Berkeley, CA 94720;

${ }^{j}$ Brown University, Providence, RI 02912;

${ }^{k}$ California Institute of Technology, Pasadena, CA 91125;

Institut d'Astrophysique Spatiale, Universite Paris-Sud, Orsay, 91405, France;

${ }^{m}$ Institute for Advanced Study, Princeton, NJ 08540

\begin{abstract}
EBEX (the E and B EXperiment) is a balloon-borne telescope designed to measure the polarisation of the cosmic microwave background radiation. During a two week long duration science flight over Antarctica, EBEX will operate 768, 384 and 280 spider-web transition edge sensor (TES) bolometers at 150, 250 and $410 \mathrm{GHz}$, respectively. The 10-hour EBEX engineering flight in June 2009 over New Mexico and Arizona provided the first usage of both a large array of TES bolometers and a Superconducting QUantum Interference Device (SQUID) based multiplexed readout in a space-like environment. This successful demonstration increases the technology readiness level of these bolometers and the associated readout system for future space missions. A total of 82, 49 and 82 TES detectors were operated during the engineering flight at 150, 250 and $410 \mathrm{GHz}$. The sensors were read out with a new SQUID-based digital frequency domain multiplexed readout system that was designed to meet the low power consumption and robust autonomous operation requirements presented by a balloon experiment. Here we describe the system and the remote, automated tuning of the bolometers and SQUIDs. We compare results from tuning at float to ground, and discuss bolometer performance during flight.
\end{abstract}

Keywords: TES, bolometer, SQUID, CMB, balloon-borne, multiplexing

Further author information:

F.A. : E-mail : francois.aubin@mail.mcgill.ca, Telephone : 15143984838

Millimeter, Submillimeter, and Far-Infrared Detectors and Instrumentation for Astronomy V, edited by Wayne S. Holland, Jonas Zmuidzinas, Proc. of SPIE Vol. 7741, 77411T

(c) 2010 SPIE · CCC code: 0277-786X/10/\$18 - doi: 10.1117/12.857596

Proc. of SPIE Vol. $774177411 \mathrm{~T}-1$ 


\section{INTRODUCTION}

The $\mathrm{E}$ and $\mathrm{B}$ experiment (EBEX) is a balloon-borne telescope designed to measure the polarisation of the cosmic microwave background (CMB) from degree to $10^{\prime}$ scales. ${ }^{1-5}$ Its primary goals are to detect or set an upper limit on the B-mode polarisation produced by inflationary gravity waves, detect B-modes generated by gravitational lensing, and characterize the properties of polarized dust. EBEX will circumnavigate Antarctica for 14 days at the end of 2011 operating 768, 384 and 280 spider-web transition edge sensor (TES) bolometers at 150, 250 and $410 \mathrm{GHz}$, respectively. These three frequency bands will allow foreground characterization and subtraction. Polarimetry is achieved with a fixed wire grid analyzer and a continuously rotating half wave plate (HWP) operating at a temperature of $4 \mathrm{~K}^{6}$

The EBEX engineering flight took place on June $11^{\text {th }} 2009$ from Fort Sumner, New Mexico. During 10 hours at an average altitude of $35,000 \mathrm{~m}$, the engineering flight allowed for the evaluation of all the major instrument subsystems, including the TES detectors, multiplexed readout and continuously rotating HWP. The left panels of Fig. 1 show sketches of the payload. ${ }^{7}$ A total of 82, 49 and 82 TES bolometers were operated at 150, 250 and $410 \mathrm{GHz}$. The detectors were read out with SQUID-based, digital frequency domain multiplexing (DfMUX) electronics, ${ }^{8}$ shown in right panel of Fig. 1 . These were developed for EBEX with the goal of achieving low power consumption, small form factor, and robust autonomous operation. ${ }^{9}$ This flight represents the first time both TES bolometers and SQUID-based multiplexed readout system have been flown on a stratospheric balloon or satellite. This demonstration increases the technology readiness level of TES bolometers and their associated readout. In this paper, we focus on the detectors and SQUID readout performance, providing an overview of the systems and an assessment of their in-flight operation.

\section{THE EBEX FOCAL PLANE}

For the science flight, EBEX will be configured with two identical focal planes, each with four $150 \mathrm{GHz}$, two $250 \mathrm{GHz}$ and one $410 \mathrm{GHz}$ TES bolometer wafers developed and fabricated at the U.C. Berkeley Microlab. A photograph of a single focal plane configured for the engineering flight with one wafer from each of the frequency bands in place is shown in the left panel of Fig. 2. Detectors are coupled to the sky by smooth-walled conical feeds. Low-pass metal-mesh filters and high-pass waveguides select the band-pass for each frequency band. A

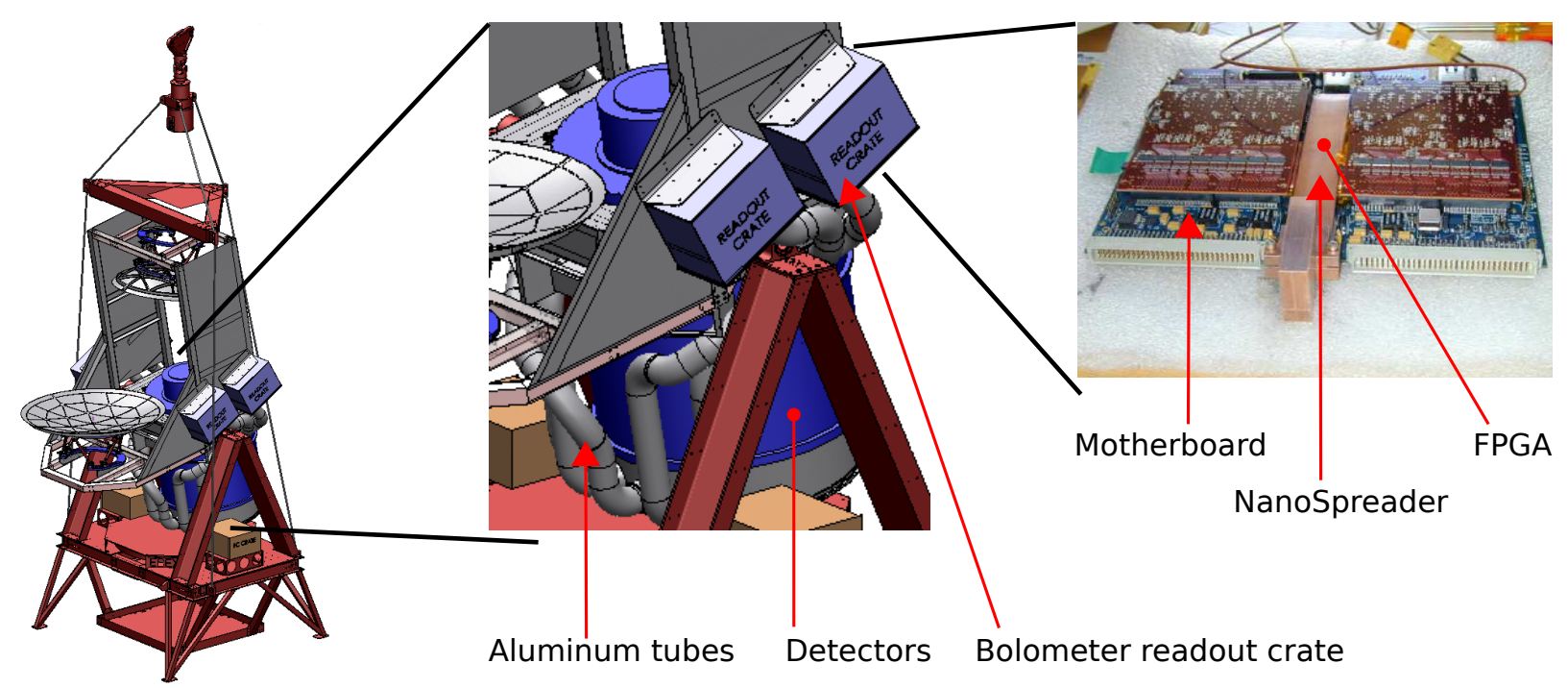

Figure 1. Drawing of the EBEX telescope and gondola (left) and zoom on the receiver (center). Detectors are located in the cryostat. Biasing and demodulating wires are routed through aluminum tubes to the bolometers readout crates. Heat from the hottest electronic part of the DfMUX readout system signal processing board (right), the FPGA, is conductively transferred to the gondola through copper commercial 'NanoSpreader' heat pipes. 


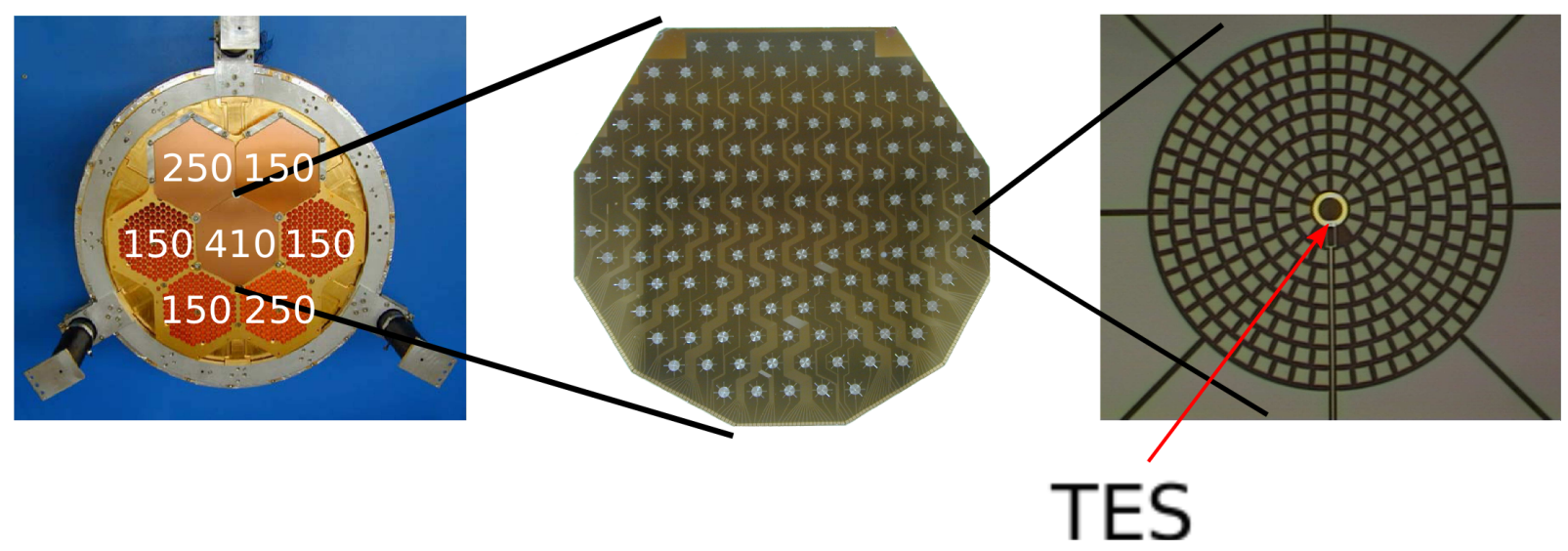

Figure 2. Photo of the EBEX focal plane (left) configured with a 150, 250 and $410 \mathrm{GHz}$ bolometer wafer for the EBEX engineering flight. The two science flight focal planes will be configured with four 150, two 250 and one $410 \mathrm{GHz}$ bolometer wafers. Low-pass filters cover conical feedhorns, only visible at unpopulated wafer positions, leading photons to the bolometer wafers through high-pass filter waveguides. An EBEX bolometer wafer (center) consists of 140 spiderweb transition edge sensor bolometers. The right panel shows a single device with TES located on the Au ring at the center of the photo.

wafer, shown in the middle panel of Fig. 2, contains 140 TES bolometers made by thin film deposition and optical lithography. A single detector is shown in the right panel of Fig. 2. A silicon nitride spider-web absorber is optimized for $\mathrm{mm}$-wavelength radiation and thermally isolated from the $270 \mathrm{mK}$ thermal bath by silicon nitride legs. An aluminum and titanium bilayer TES provides a measure of the absorber temperature. A thick layer of gold, visible at the center of the panel, increases the heat capacity of the TES, ${ }^{10}$ slowing down the TES response to a level commensurate with the absorber. Quarter-wavelength backshorts maximize the in-band photon absorption.

The EBEX detectors are being developed on the heritage from the detectors for the APEX-SZ ${ }^{11}$ and the South Pole Telescope (SPT) ${ }^{12}$ instruments. To optimize detector noise for the lower sky loading at float, the thermal conductance $(G=\partial P / \partial T)$ is reduced compared to ground-based instruments. We accomplish this by removing the gold fingers used by APEX-SZ and SPT, modifying the spider-web leg geometry and lowering the critical temperature of the TES by tuning the aluminum and titanium thicknesses. The bolometer wafers flown for the engineering flight were prototypes and their parameters had not been tuned to the design values for science grade operation. Of the three bands, the $250 \mathrm{GHz}$ detectors were found to have less susceptibility to electro-thermal oscillations at moderate detector loop gain and so we focus on these detectors only in this paper.

\section{BOLOMETER CHARACTERIZATION}

To monitor and adjust the detector fabrication process, an accurate characterization of the TES device parameters is required. Thermal conductance is determined from a measurement of the saturation power $\left(P_{s a t}\right)$ as a function of the bath temperature $\left(T_{o}\right)$ with the bolometers inside an enclosure heat sunk to $T_{o}$ so as to reduce the radiative loading to a negligible level. If the weak link thermal conductance is described by a power law,

$$
P_{\text {sat }}=\frac{C}{n+1}\left(T_{c}^{n+1}-T_{o}^{n+1}\right),
$$

where $\mathrm{n}$ and $\mathrm{C}$ are constants. ${ }^{13}$ Figure 3 shows such a measurement for bolometer 250-03-10. Fitting for $\mathrm{C}, \mathrm{n}$ and $T_{c}$, the thermal conductance can be calculated from

$$
G=C T_{c}^{n}
$$




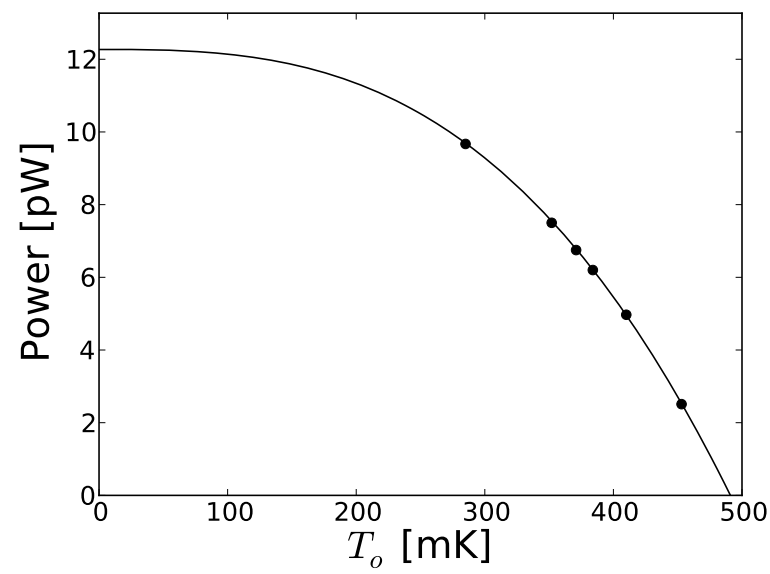

Figure 3. Saturation power as a function of the bath temperature for bolometer 250-03-10 inside an enclosure heat sunk to $T_{o}$ so as to reduce the radiative loading to a negligible level.

and the unitless factor accounting for the temperature gradient across the thermal link, ${ }^{14}$ from

$$
\gamma=\frac{(n+1)}{(2 n+3)} \frac{\left(1-\left[T_{o} / T_{c}\right]^{2 n+3}\right)}{\left(1-\left[T_{o} / T_{c}\right]^{n+1}\right)}
$$

The 49 bolometers at $250 \mathrm{GHz}$ that were operated during the engineering flight have an average critical temperature of $510 \pm 20 \mathrm{mK}$, thermal conductance of $90 \pm 10 \mathrm{pW} / \mathrm{K}$ and $\gamma$ of $0.50 \pm 0.01$.

Table 1 shows the average bolometer parameters for the $250 \mathrm{GHz}$ detectors flown in the EBEX engineering flight and target parameters for the science flight. For the engineering flight, the normal resistance $\left(R_{n}\right)$ of $1.9 \pm 0.2 \Omega$ is determined by fitting to the transfer function of the bolometer in series with an inductor and capacitor of known reactance, the optical loading $\left(P_{\text {opt }}\right)$ of $2.0 \pm 1.6 \mathrm{pW}$ is obtained from a comparison between float and dark load curves, and the bias voltage $\left(V_{b}\right)$ of $3.3 \pm 0.5 \mu V_{r m s}$ is empirically determined. The electrothermal feedback time constant $\left(\tau_{E T F}\right)$ of $13 \mathrm{~ms}$ is measured by electrically inducing a thermal signal in the TES and measuring its response as a function of frequencies. ${ }^{10}$

Table 1. Average bolometer parameters for the EBEX $250 \mathrm{GHz}$ detectors during the engineering flight and projected values for the science flight.

\begin{tabular}{|l|c|c|}
\hline Parameter & Engineering flight & Science flight \\
\hline \hline$T_{c}(\mathrm{mK})$ & 510 & 460 \\
\hline $\mathrm{G}\left(\frac{p W}{K}\right)$ & 90 & 67 \\
\hline$\gamma$ & 0.50 & 0.50 \\
\hline$R_{n}(\Omega)$ & 1.9 & 1.0 \\
\hline$P_{\text {opt }}(\mathrm{pW})$ & $2.0 *$ & 3.5 \\
\hline$V_{b}\left(\mu V_{r m s}\right)$ & 3.3 & 1.8 \\
\hline$\tau_{E T F}(\mathrm{~ms})$ & 13 & 10 \\
\hline
\end{tabular}

* A neutral density filter was in place to attenuate the optical loading during the engineering flight. 


\section{THE READOUT SYSTEM}

Readout signal multiplexing reduces the heat load on the sub-Kelvin bolometer stage and is essential for meeting the cryogenic heat load budget when operating large arrays of sub-Kelvin detectors. EBEX uses frequency domain multiplexing ${ }^{15}$ to transmit signals from several bolometers on a single set of wires, referred to as a multiplexed module or 'mux', from the sub-Kelvin bolometer stage to a SQUID mounted at $4 \mathrm{~K} .{ }^{16}$ Within each multiplexed module, each detector is electrically biased in its superconducting transition with a unique sinusoidal voltage bias carrier in the frequency range of $200 \mathrm{kHz}$ to $1.2 \mathrm{MHz}$, selected by a inductor-capacitor bandpass filter in series with the TES. A multiplexing factor of eight bolometers per SQUID was used during the engineering flight. We are planning to implement a multiplexing factor of 12 for the science flight.

The conceptual circuit of one multiplexer module of the DfMUX is shown in Fig. 4. The constant amplitude sinusoidal voltage biases for a single multiplexed module are synthesized as a comb by the room temperature electronics ("Carrier" in Fig. 4) and transmitted to the sub-Kelvin detector stage. The TES resistance is amplitude modulated by radiation from the sky, encoding the signal in the carrier's sideband through negative electro-thermal feedback. ${ }^{17}$ A signal appears in the bolometer as a current inversely proportional to the incident optical power. The current from all the carriers in a multiplexed module are summed at the input to the SQUID together with a comb of nuller sinusoids ("Nuller" in Fig. 4) that are $180^{\circ}$ out of phase with the carrier signals. These nulling signals drastically reduce the dynamic range requirements of the SQUID by removing the carrier signal without affecting its sidebands. The nulled signal comb is transmitted to the ambient temperature DfMUX electronics where the entire amplitude modulated and nulled comb is digitized at $25 \mathrm{MHz}$ ("Demod" in Fig. 4). Firmware algorithms implemented on a Xilinx Field-Programmable Gate Array (FPGA) lock-in demodulate each detector's sky signal separately, down sampling them to $190.73 \mathrm{~Hz}$. The bolometer signals are transmitted over Ethernet to the flight control computer where they are recorded on disk. ${ }^{3}$

The total power budget for a balloon payload is limited to $\sim 1 \mathrm{~kW}$. The digital version of the analog frequency multiplexing electronics was developed to fulfill this constraint. During the engineering flight, 12 DfMUX boards used a total of $240 \mathrm{~W}$, or $20 \mathrm{~W}$ per DfMUX board. For the science flight, 28 DfMUX boards are planned. The multiplexing factor improvement increases the power consumption to $21 \mathrm{~W}$ per DfMUX board for a total expected power consumption of $580 \mathrm{~W}$.

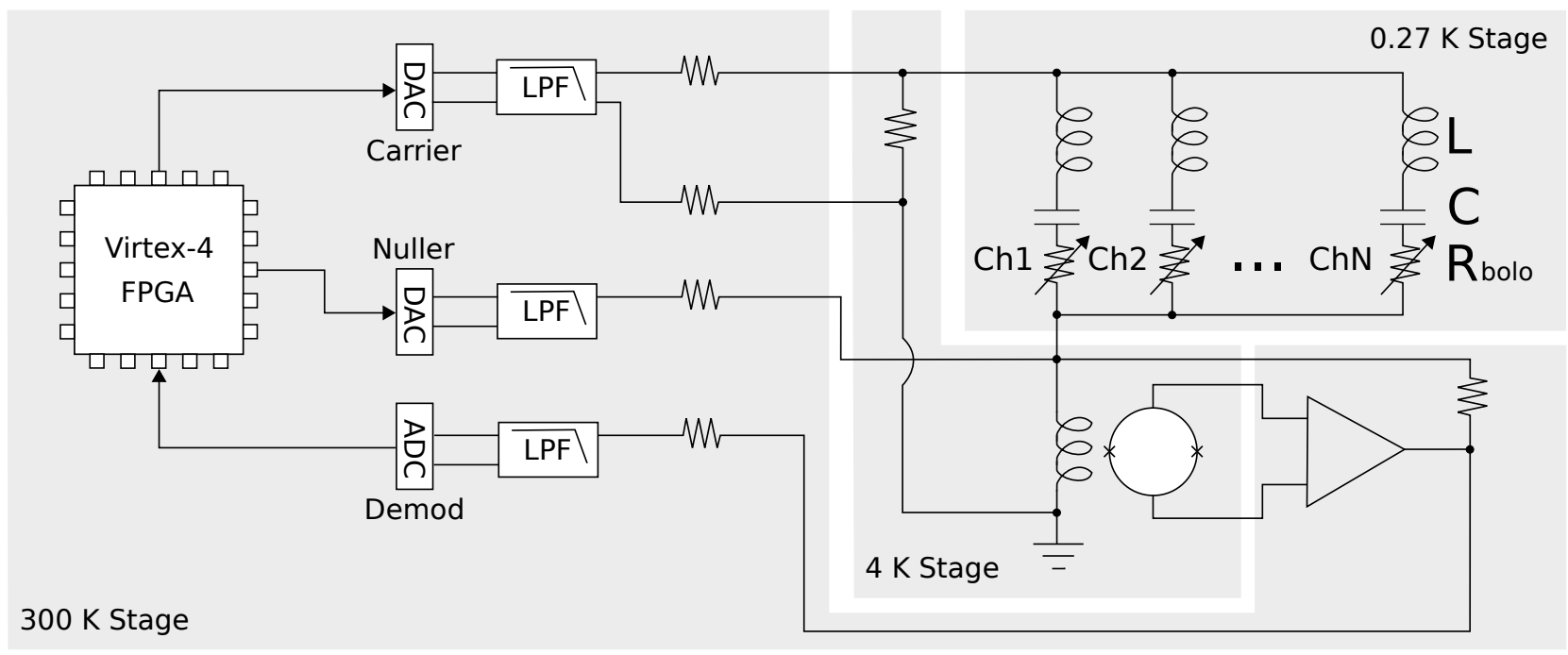

Figure 4. Simplified schematic showing the digital frequency domain multiplexed readout for a single detector module. The FPGA synthesizes a carrier comb of sinusoids that bias the detectors. Each detector is placed in series with an inductor and capacitor on the sub-Kelvin stage which selects a single bias carrier from the comb. The sky signal amplitude modulates the bias carrier. The bias carriers from all detectors in a module are summed with a nuller comb at the input of the SQUID coil. The signals are amplified by the SQUID and digitized before being demodulated in the FPGA. 
Cooling on a balloon platform must be achieved through radiation. Heat is removed from the boards using commercial 'NanoSpreader' heat pipes ${ }^{18}$ that are glued to the hottest components on one end and attached mechanically to a radiating back plane on the other as shown in Fig. 1. In addition, the backplane also conducts heat to the gondola body, which also serves as a radiator. For the Antarctic long duration flight, for which there is higher power density in the readout crates, we will implement a liquid coolant system to aid in distributing the dissipated heat over a larger surface area of the gondola.

The bolometer readout crates, mounted on the sides of the gondola, are electrically isolated from the gondola to minimize electrical pickup. Radio frequency gaskets and aluminum tape are used at the crate seams to provide an RF seal. Fiber optic ports are used for Ethernet connections to the crates. The readout crates are electrically isolated from the flight computer and other readout crates by RF-filters. All signals going to the cryostat are routed through aluminum tubes and connect directly to the RF sealed cryostat through RF-filters.

\section{BOLOMETER PERFORMANCE}

\subsection{Noise Expectation}

Table 2 shows the expected white noise contributions from different sources referred to the current noise for an amplitude modulated signal through the SQUID input coil. Here, $\mathrm{k}$ is the Boltzmann constant, $R_{\text {bolo }}$ is the bolometer resistance, $\mathrm{h}$ is the Planck constant and $\Delta \nu$ is the bandwidth of the absorbed photons $(70 \mathrm{GHz}$ for the EBEX $250 \mathrm{GHz}$ band). Separate columns show the expectation for average parameters of the $250 \mathrm{GHz}$ detectors flown during the engineering flight and for the target $250 \mathrm{GHz}$ detector design parameters of the science flight. For noise sources such as phonon and photon noise that produce thermal power on the detectors, we refer the noise equivalent power (NEP) to a current through the SQUID coil by multiplying by the AC-biased TES responsivity $\sqrt{2} / V_{b}^{R M S}$. A factor $\sqrt{2}$ is included for noise sources that produce uncorrelated power on both sides of the bias carrier to account for the demodulation which maps both sidebands to the same frequency at baseband. This is the case for readout noise and bolometer Johnson noise. ${ }^{10}$ We leave this factor explicit in the Table 2 to emphasize that this factor manifests itself post-demodulation and is not physically present at the SQUID input.

Table 2. Expected sources of noise in EBEX for the $250 \mathrm{GHz}$ detectors during the engineering flight and projected for the science flight. No electro-thermal feedback suppression factor is included for Johnson noise in this table because the engineering flight detectors were not operated with high loop gain.

\begin{tabular}{|l|c|c|c|}
\hline Noise source & Equation & $\begin{array}{c}\text { Engineering flight } \\
\left(\mathrm{pA}_{r m s} / \sqrt{\mathrm{Hz}}\right)\end{array}$ & $\begin{array}{c}\text { Science flight } \\
\left(\mathrm{pA}_{r m s} / \sqrt{\mathrm{Hz}}\right)\end{array}$ \\
\hline \hline 4 K Readout & $\mathrm{N} / \mathrm{A}$ & $\sqrt{2} \cdot 3.4$ & $\sqrt{2} \cdot 4.5$ \\
\hline Warm Readout & $\mathrm{N} / \mathrm{A}$ & $\sqrt{2} \cdot 5.7$ & $\sqrt{2} \cdot 4.8$ \\
\hline Bolometer Johnson & $\sqrt{2} \cdot \sqrt{\frac{4 k T_{c}}{R_{\text {bolo }}}}$ & $\sqrt{2} \cdot 5.0$ & $\sqrt{2} \cdot 6.0$ \\
\hline Bolometer phonon & $\frac{\sqrt{2}}{V_{b}} \cdot \sqrt{\gamma 4 k T_{c}^{2} G}$ & 10.9 & 15.4 \\
\hline Bolometer photon & $\frac{\sqrt{2}}{V_{b}} \cdot \sqrt{2 P_{o p t} h \nu}$ & 11.0 & 25.2 \\
\hline Bolometer photon bunching & $\frac{\sqrt{2}}{V_{b}} \cdot \sqrt{\frac{P_{o p t}^{2}}{\Delta \nu}}$ & 3.3 & 9.2 \\
\hline Total & & 19.7 & 33.4 \\
\hline
\end{tabular}

\subsection{SQUID and Readout Noise}

In addition to the detector readout channels, we record signals from 'dark' SQUIDs that were not connected to any detectors. Noise from the 'dark' SQUIDs was demodulated at eight frequencies from 200 to $900 \mathrm{kHz}$, the frequency range used to bias the bolometers. Figure 5 shows an example of the signal power spectrum recorded for SQUID b05-A3 at $199836 \mathrm{~Hz}$. The expected 'dark' SQUID readout noise is slightly smaller then 


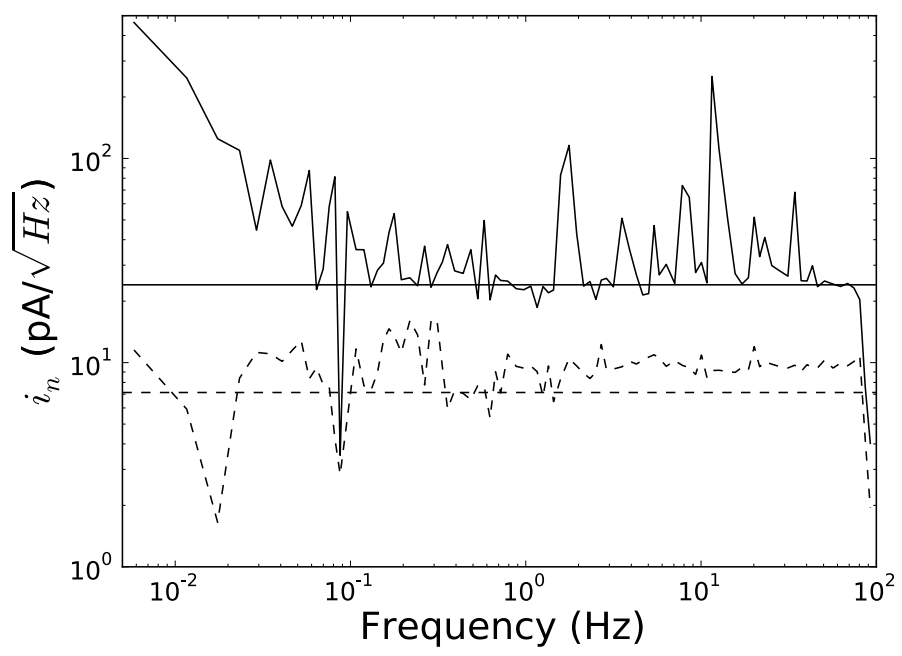

Figure 5. Current density spectrum of SQUID b05-A3 at $199836 \mathrm{~Hz}$ (dashed line) and bolometer 250-03-10 (solid line) operated at $70 \%$ of its normal resistance during the EBEX engineering flight. Peaks at harmonics of $2 \mathrm{~Hz}$ are known optical signals from the half-wave plate that are not related to the performance of the detector or readout system. Horizontal lines show expectations based on Table 2 .

the readout term in Tab. 2 because 'dark' SQUID channels have negligible contributions from the carrier and nuller synthesis electronics. The spectrum is white with an average value of $8.9 \mathrm{p} A_{r m s} / \sqrt{H z}$, averaged between 2.2 and $2.7 \mathrm{~Hz}$ where no undesired optical signal is present in the bolometer signal. This is about $20 \%$ higher than the expected value, consistent with the noise levels for six of the demodulated frequencies. We find most dark SQUID channels show noise higher than expected on the same order as the one shown in Fig. 5. The cause of the excess noise is under investigation. A copy of the SQUIDs and readout system operating in a lab cryostat exhibit noise consistent with the expected value. The observed SQUID readout noise has been taken into account when calculating expected bolometer noise.

\subsection{Noise With Detectors Above the Superconducting Transition}

One of the early steps in tuning the bias voltages for the detectors is to 'over-bias' them by providing enough electrical bias power to keep them above the superconducting transition. The detectors are normally 'over-biased' while the sub-Kelvin detector stage is cooling to its nominal operating temperature. After that temperature is reached the electrical bias power is decreased to lower the detectors into their transition.

'Over-biased' detector noise provides a measurement of the end-to-end readout system noise excluding phonon and photon noise from the TESs, since the detectors behave approximately as resistors. Figure 6 shows the distribution of the measured to predicted noise ratios for the $250 \mathrm{GHz}$ detectors from the receiver sub-system alone closed to light, measured post-flight. The average measured to predicted ratio is $1.0 \pm 0.1$, excluding outliers.

\subsection{In Transition Noise}

Figure 5 shows the noise spectrum of detector 250-03-10 during the EBEX engineering flight biased to $70 \%$ of the normal resistance of the bolometer. The measured white noise level is $23 \mathrm{pA}_{r m s} / \sqrt{H z}$, consistent with the expected noise level. The bolometer parameters are thermal conductance of $72 \mathrm{pW} / \mathrm{K}$, critical temperature of $490 \mathrm{mK}$, normal resistance of $1.5 \Omega$ and voltage bias of $2.6 \mu V_{r m s}$ at $381888 \mathrm{~Hz}$. Optical loading on the detector is measured to be $2.3 \mathrm{pW}$. Peaks at harmonics of $2 \mathrm{~Hz}$ are well understood optical signals from the half-wave plate that are not related to the performance of the detector or the readout system. While this detector belongs to a group of detectors showing nominal noise, there are others with noise level that is typically a factor of 1.3 


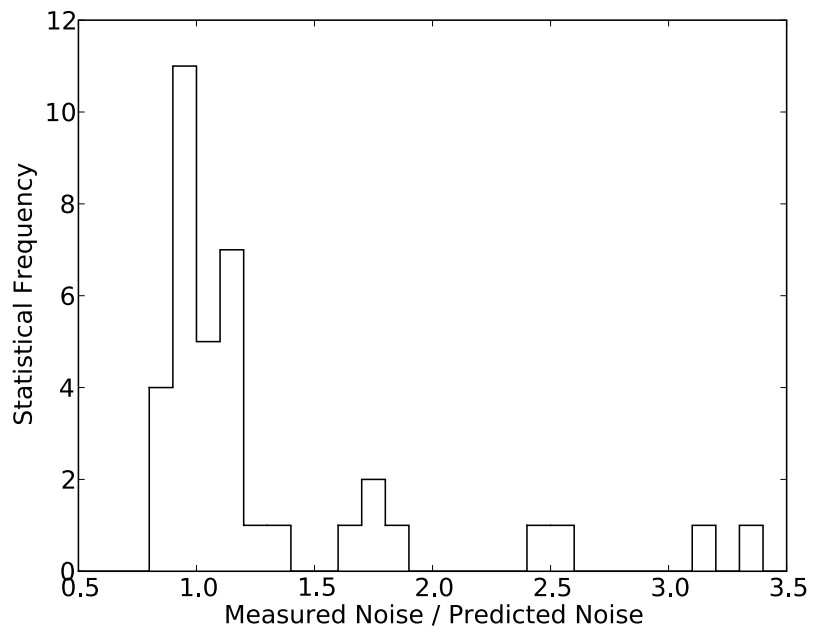

Figure 6. Distribution of the measured to predicted noise ratio for the $250 \mathrm{GHz}$ bolometers electrically biased above their superconducting transition measured, with the receiver alone on the ground.

higher than expectations. Similar noise degradation can be recreated in the lab when a SQUID's dynamic range is used up by signals picked up from unstable detectors that are oscillating, such as the ones operating during the flight at other frequency bands. This is under investigation.

\section{IN FLIGHT TUNING}

EBEX was launched with the SQUIDs tuned and detectors 'over-biased' to maximise observation time. During the ascent, an event of unknown origin caused spurious magnetic flux to be trapped by the SQUIDs. Though this event wasn't forseen, it allowed for a demonstration of the automated remote setup and tuning of the bolometer camera. This involved releasing the trapped SQUID flux by heating the devices above their superconducting transition and recooling them, warming the detectors above their transition, 'over-biasing' the detectors, nulling the carriers, cooling the detector stage, then lowering the detectors into their transitions. A total of 82 minutes elapsed for retuning the entire focal plane, excluding the time for re-cooling to operating temperatures. This is longer than the time reported in Ref. 9, because a longer version of the tuning was employed to map out the parameter space of the SQUID and bolometer bias values. The left panel of Fig. 7 shows the voltage as a function of the flux bias for the SQUID tuning procedure of b01-B3 executed at float and on the ground. Though the SQUIDs typically required a different tuning at float, there was no degradation in their peak-to-peak response function. The right panel of Fig. 7 shows the bolometer tuning current as a function of the voltage bias for bolometer 250-09-03 repeatedly executed at various payload altitudes to measure optical loading.

\section{CONCLUSION}

Transition edge sensor bolometers with a multiplexed SQUID readout have been operated from a balloon platform for the first time during the EBEX engineering flight in June 2009. The digital frequency domain readout electronics designed for EBEX successfully tuned, monitored and operated the TES detectors and SQUIDs in a space-like environment. We have achieved bolometer sensitivity at the expected level for a subset of the detector channels. This demonstration increases the technology readiness level for TES bolometers and the DfMUX SQUID readouts for satellite applications. We are now upgrading and fully populating the EBEX focal plane for the Antarctic science flight. 

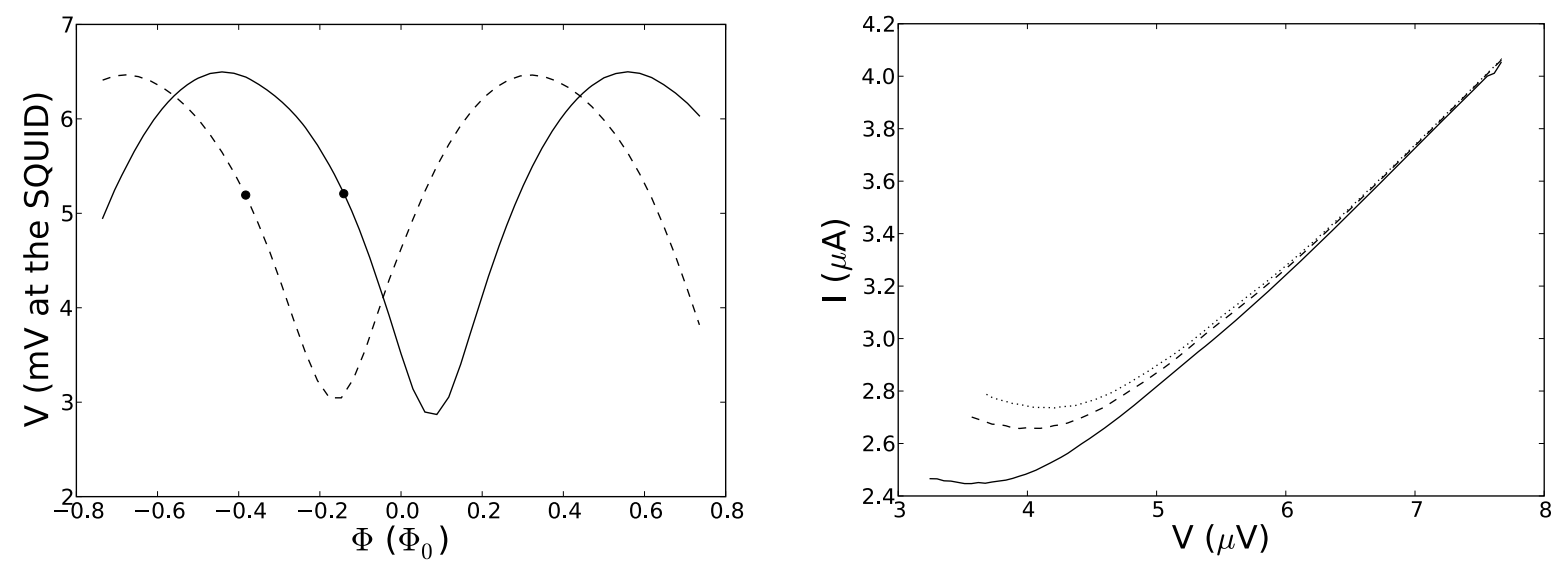

Figure 7. Results of in flight tuning of the focal plane. Left : Voltage vs magnetic flux response for SQUID b01-B3 on the ground (solid line) and in flight (dashed line). Chosen SQUID bias points are marked with circles. A difference in the magnetic environment is evident by the shift in the response. Right : Current as a function of voltage for bolometer 250-09-03 at altitude $18287 \mathrm{~m}$ (solid line), 21,403 $\mathrm{m}$ (dashed line) and 24,964 $\mathrm{m}$ (dotted line).

\section{ACKNOWLEDGMENTS}

EBEX is a NASA supported mission through grants number NNX08AG40G and NNX07AP36H. We also acknowledge support from French Centre national de la recherche scientifique (CNRS), the UK Science and Technology Facilities Council (STFC). This project makes use of the Minnesota Supercomputing Institute and the National Energy Research Scientific Computing Center (NERSC) supported by the office of Science of the U.S. Department of Energy under contract No. DE-AC02-05CH11231. The McGill authors acknowledge funding from the Canadian Space Agency (CSA), Natural Sciences Engineering Research Council (NSERC), Canadian Institute for Advanced Research (CIFAR), Canadian Foundation fo Innovation (CFI) and Canada Research Chairs program (CRC). MD acknowledges support from an Alfred P. Sloan Research Fellowship and FA from Fonds de recherche sur la nature et les technologies (FQRNT).

We thank Columbia Scientific Balloon Facility for their enthusiastic support of EBEX. The McGill team thanks Martin Lueker, Bryan Steinbach and Zigmund Kermish for valuable discussions and the POLARBEAR team for valuable contributions to the DfMux readout and software development.

\section{REFERENCES}

[1] Reichborn-Kjennerud, B. et al., "EBEX: A Balloon-Borne CMB Polarization Experiment," Proc. SPIE 7741 (2010).

[2] Sagiv, I. et al., "The EBEX Cryostat and Supporting Electronics," Proceedings of the Twelfth Marcel Grossman Meeting (2010).

[3] Milligan, M. et al., "Software systems for operation, control, and monitoring of the ebex instrument," Proc. SPIE $\mathbf{7 7 4 0}$ (2010).

[4] Grainger, W. et al., "EBEX: the E and B Experiment," Proc. SPIE 7020, 70202N (2008).

[5] Oxley, P. et al., "The EBEX Experiment," Proc. SPIE 5543, 320-331 (2004).

[6] Hanany, S., Hubmayr, J., Johnson, B. R., Matsumura, T., Oxley, P., and Thibodeau, M., "Millimeter-wave achromatic half-wave plate," Appl. Opt. 44, 4666-4670 (2005).

[7] Reichborn-Kjennerud, B., [Building and Flying the E and B Experiment to Measure the Polarization of the Cosmic Microwave Background], Ph.D. thesis, Columbia University (2010).

[8] Dobbs, M., Bissonnette, E., and Spieler, H., "Digital Frequency Domain Multiplexer for MillimeterWavelength Telescopes," IEEE Transactions on Nuclear Science 55, 21-26 (2008). 
[9] MacDermid, K., Hyland, P., Aubin, F., Bissonnette, E., Dobbs, M., Hubmayr, J., Smecher, G., and Wairrach, S., "Tuning of Kilopixel Transition Edge Sensor Bolometer Arrays with a Digital Frequency Multiplexed Readout System," AIP Conf. Proc. 1185, 253-256 (2009).

[10] Hubmayr, J., Aubin, F., Bissonnette, E., Dobbs, M., Hanany, S., Lee, A., MacDermid, K., Meng, X., Sagiv, I., and Smecher, G., "Design and characterisation of TES bolometers and SQUID readout electronics for a balloon-borne application," Proc. SPIE 7020, 70200J (2008).

[11] Mehl, J. et al., "TES Bolometer Array for the APEX-SZ Camera," Journal of Low Temperature Physics 151, 697-702 (2008).

[12] Shirokoff, E. et al, "The South Pole Telescope SZ-Receiver Detectors," IEEE Transactions on Applied Superconductivity 19, 517-519 (2009).

[13] Hubmayr, J., [Bolometric detectors for EBEX : a balloon-borne cosmic microwave background polarimeter], Ph.D. thesis, University of Minnesota (2009).

[14] Mather, J., "Bolometer noise: nonequilibrium theory," Appl. Opt. 21(6), 1125 (1982).

[15] Lanting, T. M., Cho, H., Clarke, J., M, Dobbs, Lee, A., Richards, P. L., Smithm, A. D., and Spieler, H. G., "A frequency-domain squid multiplexer for arrays of transition-edge superconducting sensors," IEEE Trans. Appl. Sup. 13(2), 626 (2003).

[16] Huber, M. E., Neil, P. A., Benson, R. G., Burns, D. A., Corey, A. M., Flynn, C. S., Kitaygorodskaya, Y., Massihzadeh, O., Martinis, J. M., and Hilton, G. C., "DC SQUID serie array amplifiers with $120 \mathrm{MHz}$ bandwidth (corrected)," IEEE Transactions on Applied Superconductivity 11, 4048-4053 (2001).

[17] Irwin, K. D., Hilton, G. C., Wollman, D. A., and Martinis, J. M., "Thermal-response time of superconducting transition-edge microcalorimeters," J. Appl. Phys. 83, 3978-3985 (1998).

[18] Celsia http://www.celsiatechnologies.com/ (2006). 